\title{
An Exponential Spline Approach to the Generalized Sine-Gordon Equation
}

\author{
Reza Mohammadi* \\ Department of Mathematics, University of Neyshabur, Postal code 91136-899 Neyshabur, Iran. \\ * Corresponding author. Tel.: +985142613311; email: rez.mohammadi@gmail.com \\ Manuscript submitted March28, 2015; accepted October 24, 2015. \\ doi: 10.17706/ijapm.2015.5.4. 259-266
}

\begin{abstract}
The nonlinear sine-Gordon equation is used to model many nonlinear phenomena. Numerical simulation of the solution to the one-dimensional generalized sine-Gordon equation is considered here. Two implicit three time-level difference schemes are developed, by using the exponential spline function approximation. We consider both Dirichlet and Neumann boundary conditions. The resulting spline difference schemes are analyzed for local truncation error, stability and convergence. It has been shown that by suitably choosing the parameters, we can obtain two schemes of $O\left(k^{2}+k^{2} h^{2}+h^{2}\right)$ and $O\left(k^{2}+k^{2} h^{2}+h^{4}\right)$. In the end, some numerical examples are provided to demonstrate the effectiveness of the proposed schemes.
\end{abstract}

Key words: Exponential spline, finite difference, generalizedsine-gordon equation, dirichlet and neumann boundary conditions, stability analysis, convergence.

\section{Introduction}

This paper is devoted to the numerical computation of the one-dimensional time-dependent nonlinear sine-Gordon equation. The initial-value problem of the one-dimensional generalized sine-Gordon equation is given by the following equation:

$$
u_{t t}+\rho u_{t}=u_{x x}-f(x, t, u), \quad x \in(a, b), \quad t>t_{0},
$$

subjected to the initial conditions

$$
u\left(x, t_{0}\right)=\phi(x), \quad u_{t}\left(x, t_{0}\right)=\psi(x), \quad x \in[a, b]
$$

and with following Dirichletboundary conditions:

$$
u(a, t)=g_{0}(t), \quad u(b, t)=g_{1}(t), \quad t \geq t_{0},
$$

where the functions $\phi(x)$ and $\psi(x)$ are wave modes or kinks and velocity, respectively. The parameter $\rho$ is the so-called dissipative term, which is assumed to be a real number with $\rho \geq 0$ [1], [2]. We shall assume that $\phi(x), \psi(x), g_{0}(t)$ and $g_{1}(t)$ are continuously differentiable up to order 2 .

Klein-Gordon and sine-Gordon equations have applications in various research areas, such as differential 
geometry and relativistic field theory [3]-[9].

The numerical solutions to the nonlinear sine-Gordon equation have received considerable attention in the literature (see [10-16]). Mohammadi [17]-[19] developed different spline schemes to find the numerical solution of different type of partial differential equations.

In this paper we have developed a three time-level implicit method by using the exponential spline function for solution of the nonlinear partial differential equation (1). The method involves some parameters, and its order can be increased from $O\left(k^{2}+k^{2} h^{2}+h^{2}\right)$ to $O\left(k^{2}+k^{2} h^{2}+h^{4}\right)$ by an appropriate choice of the parameters.

\section{Proposition of the Method}

The domain $[a, b] \times[0, T]$ is divided to a $n \times m$ mesh with the special step size $h=(b-a) / n$ in $x$ direction and time step size $k=T / m$, respectively. Denote

$$
\Omega_{h}=\left\{x_{l}=a+l h, l=0,1, \ldots, n\right\}, \quad \Omega_{k}=\left\{t_{j}=t_{0}+j k, j=0,1, \ldots, m\right\} .
$$

in which $n$ and $m$ are integers. The notation $u_{l}^{j}$ be a grid function on $\Omega_{h} \times \Omega_{k}$, which is used for the difference approximation of $u(l h, j k)$. A function $S\left(x, t_{j}\right)$ of class $C^{2}[a, b]$ which interpolates $u\left(x, t_{j}\right)$ at the mesh points $\left(x_{l}, t_{j}\right)$, depends on a parameter $\tau$, reduces to cubic spline $S\left(x, t_{j}\right)$, in $[a, b]$ as $\tau \rightarrow 0$, is termed an exponential spline function. For each segment $\left[x_{l}, x_{l+1}\right], l=0,1, \ldots, n-1$ the function $S\left(x, t_{j}\right)$, can be defined in the following form

$$
S\left(x, t_{j}\right)=a_{l}^{*}\left(t_{j}\right)+b_{l}^{*}\left(t_{j}\right)\left(x-x_{l}\right)+c_{l}^{*}\left(t_{j}\right) e^{i \tau\left(x-x_{l}\right)}+d_{l}^{*}\left(t_{j}\right) e^{-i \tau\left(x-x_{l}\right)},
$$

where $a_{l}^{*}\left(t_{j}\right), b_{l}^{*}\left(t_{j}\right), c_{l}^{*}\left(t_{j}\right)$ and $d_{l}^{*}\left(t_{j}\right)$ are unknown coefficients, $\tau$ is a free parameter and $i=\sqrt{-1}$. We first develop the explicit expressions for the four coefficients in (5) in terms of $u_{l}^{j}, u_{l+1}^{j}$, $M_{l}^{j}$ and $M_{l+1}^{j}$. We can determine the four unknown coefficients in (5) as

$$
a_{l}^{*}\left(t_{j}\right)=u_{l}^{j}+\frac{M_{l}^{j}}{\tau^{2}}, b_{l}^{*}\left(t_{j}\right)=\frac{M_{l+1}^{j}-M_{l}^{j}+\tau^{2}\left(u_{l+1}^{j}-u_{l}^{j}\right)}{h \tau^{2}}, c_{l}^{*}\left(t_{j}\right)=\frac{M_{l}^{j}-e^{i \tau h} M_{l+1}^{j}}{\tau^{2}\left(e^{2 i \tau h}-1\right)}, d_{l}^{*}\left(t_{j}\right)=\frac{e^{i \tau h}\left(M_{l+1}^{j}-e^{i \tau h} M_{l}^{j}\right)}{\tau^{2}\left(e^{2 i \tau h}-1\right)} .
$$

The continuity of the first derivative of $S\left(x, t_{j}\right)$ at $\left(x_{l}, t_{j}\right)$ yields the following consistency relation:

$$
u_{l+1}^{j}-2 u_{l}^{j}+u_{l-1}^{j}=h^{2}\left(\lambda_{1} M_{l-1}^{j}+2 \lambda_{2} M_{l}^{j}+\lambda_{1} M_{l+1}^{j}\right)
$$

where

$$
\lambda_{1}=(\theta \csc \theta-1) / \theta^{2}, \quad \lambda_{2}=(1-\theta \cot \theta) / \theta^{2}, \quad \theta=\tau h, \quad M_{l}^{j}=S^{\prime \prime}\left(x_{l}, t_{j}\right) \quad l=0,1, \ldots, n \text { and } j=0,1, \ldots, m
$$

Similar to $(6)$ for the $(j+1)$ th and $(j-1)$ th time levels we have 


$$
u_{l+1}^{j \pm 1}-2 u_{l}^{j \pm 1}+u_{l-1}^{j \pm 1}=h^{2}\left(\lambda_{1} M_{l-1}^{j \pm 1}+2 \lambda_{2} M_{l}^{j \pm 1}+\lambda_{1} M_{l+1}^{j \pm 1}\right)
$$

We develop an approximation for (1) in which the time derivative is replaced by following finite difference approximation

$$
{\overline{u_{t t l}}}^{j}=\left(u_{l}^{j+1}-2 u_{l}^{j}+u_{l}^{j-1}\right) / k^{2}=u_{t t l}^{j}+O\left(k^{2}\right), \quad \bar{u}_{t l}^{j}=\left(u_{l}^{j+1}-u_{l}^{j-1}\right) / 2 k=u_{t l}^{j}+O\left(k^{2}\right),
$$

and the space derivative by the non-polynomial spline function approximation

$$
u_{x x l}^{j}=S^{\prime \prime}\left(x_{l}, t_{j}\right)=M_{l}^{j}+O\left(h^{2}\right)
$$

To develop a new approximation for (1) the sine-Gordon equation (1) is then replaced by

$$
\left[\left(u_{l}^{j+1}-2 u_{l}^{j}+u_{l}^{j-1}\right) / k^{2}\right]+\left[\rho\left(u_{l}^{j+1}-u_{l}^{j-1}\right) /(2 k)\right]+f_{l}^{j}=\sigma M_{l}^{j-1}+(1-2 \sigma) M_{l}^{j}+\sigma M_{l}^{j+1}
$$

where $0 \leq \sigma \leq 1, f_{l}^{j}=f\left(x_{l}, t_{j}, u_{l}^{j}\right)$ and $l=0,1, \ldots, n, j \geq 0$.

The addition of (6) multiplied by $(1-2 \sigma)$ to (7) multiplied by $\sigma$ gives

$$
\begin{aligned}
& \lambda_{1}\left(\sigma M_{l-1}^{j-1}+(1-2 \sigma) M_{l-1}^{j}+\sigma M_{l-1}^{j+1}\right)+2 \lambda_{2}\left(\sigma M_{l}^{j-1}+(1-2 \sigma) M_{l}^{j}+\sigma M_{l}^{j+1}\right)+ \\
& \lambda_{1}\left(\sigma M_{l+1}^{j-1}+(1-2 \sigma) M_{l+1}^{j}+\sigma M_{l+1}^{j+1}\right)=\delta_{x}^{2}\left(\sigma u_{l}^{j-1}+(1-2 \sigma) u_{l}^{j}+\sigma u_{l}^{j+1}\right) / h^{2},
\end{aligned}
$$

where $\delta_{x}$ is the central difference operator with respect to $x$ so that $\delta_{x}^{2} u_{l}^{j}=u_{l-1}^{j}-2 u_{l}^{j}+u_{l+1}^{j}$.

After the elimination of the $M$ 's in (11) by means of (10) we have

$$
\begin{gathered}
\left(\lambda_{1}(1-(k \rho / 2))-\sigma \mu^{2}\right) u_{l-1}^{j-1}+2\left(\lambda_{2}(1-(k \rho / 2))+\sigma \mu^{2}\right) u_{l}^{j-1}+\left(\lambda_{1}(1-(k \rho / 2))-\sigma \mu^{2}\right) u_{l+1}^{j-1}- \\
\left(2 \lambda_{1}+(1-2 \sigma) \mu^{2}\right) u_{l-1}^{j}-2\left(2 \lambda_{2}+(1-2 \sigma) \mu^{2}\right) u_{l}^{j}-\left(2 \lambda_{1}+(1-2 \sigma) \mu^{2}\right) u_{l+1}^{j}+ \\
\left(\lambda_{1}(1+(k \rho / 2))-\sigma \mu^{2}\right) u_{l-1}^{j+1}+2\left(\lambda_{2}(1+(k \rho / 2))-\sigma \mu^{2}\right) u_{l}^{j+1}+\left(\lambda_{1}(1+(k \rho / 2))-\sigma \mu^{2}\right) u_{l+1}^{j-1}+ \\
k^{2}\left(\lambda_{1} f_{l-1}^{j}+2 \lambda_{2} f_{l}^{j}+\lambda_{1} f_{l+1}^{j}\right)=0, \quad l=1,2, \ldots, n-1,
\end{gathered}
$$

where $\mu=k / h$ is the mesh ratio, $\lambda_{1}$ and $\lambda_{2}$ are parameters defined in (6).

So by expanding (12) in Taylor series in terms of $u\left(x_{l}, t_{j}\right)$ and it's derivatives, and replacing the derivatives involving $t$ by the relation

$$
\partial u^{l+j} / \partial x^{l} \partial t^{j}=-\partial u^{l+2 j} / \partial x^{l+2 j}
$$

we obtain the local truncation error. The principal part of the local truncation error of the method is

$$
\begin{aligned}
T_{l}^{j}=\{ & \left\{2\left(\lambda_{1}+\lambda_{2}\right)-1\right] u_{x x}+h^{2}\left(-1 / 12+\lambda_{1}\right) \partial^{4} / \partial x^{4}-\left[h^{4}\left(1-30 \lambda_{1}\right) / 360-k^{2}\left(\rho\left(\lambda_{1}+\lambda_{2}\right)-3 \sigma\right) / 3\right] \times \\
& \left.\partial^{6} / \partial x^{6}-(1 / 20160)\left[h^{6}\left(1-56 \lambda_{1}\right)+3360 k^{2}\left(\lambda_{1}+\lambda_{2}\right)+1680 k^{2} h^{2}\left(2 \lambda_{1} \rho-\sigma\right)\right] \partial^{8} / \partial x^{8}+\ldots\right\} u_{l}^{j},
\end{aligned}
$$

which tends to zero as $h$ and $k$ tend to zero simultaneously, so method is consistent with (1). 
By choosing suitable values of parameters $\lambda_{1}$ and $\lambda_{2}$ in (12) we obtain the following classes of methods:

- If we choose $\lambda_{1}+\lambda_{2}=1 / 2$ and $\lambda_{1} \neq 1 / 12$ in (12) we obtain various schemes of $O\left(k^{2}+k^{2} h^{2}+h^{2}\right)$.

- If we choose $\lambda_{1}=1 / 12$ and $\lambda_{2}=5 / 12$, in (12) we obtain a new scheme of $O\left(k^{2}+k^{2} h^{2}+h^{4}\right)$.

\section{Stability and Convergence Analysis}

In this section, we will discuss the stability and convergence of the scheme (12) for numerical solution of Dirichlet boundary problem (1)-(3), with homogenous conditions $g_{0}(t)=g_{1}(t)=0$. First we define the following difference operators

$$
\begin{aligned}
& \delta_{2 t} u_{l}^{j}=u_{l}^{j+1}-u_{l}^{j-1}, \quad \delta_{t}^{+} u_{l}^{j}=u_{l}^{j+1}-u_{l}^{j}, \quad \delta_{t}^{-} u_{l}^{j}=u_{l}^{j}-u_{l}^{j-1}, \quad \delta_{2 t} u_{l}^{j}=\left(\delta_{t}^{+}+\delta_{t}^{-}\right) u_{l}^{j}, \quad \delta_{t}^{2} u_{l}^{j}=\left(\delta_{t}^{+}-\delta_{t}^{-}\right) u_{l}^{j}, \\
& \delta_{x}^{+} u_{l}^{j}=u_{l+1}^{j}-u_{l}^{j}, \quad \delta_{x}^{-} u_{l}^{j}=u_{l}^{j}-u_{l-1}^{j}, \quad \delta_{x}^{2} u_{l}^{j}=u_{l+1}^{j}-2 u_{l}^{j}+u_{l-1}^{j}, \quad\left(u_{t}\right)_{l}^{j}=\delta_{t}^{+} u_{l}^{j} / k, \quad\left(u_{x}\right)_{l}^{j}=\delta_{x}^{+} u_{l}^{j} / h .
\end{aligned}
$$

If $v=\left(v_{0}, v_{1}, \ldots, v_{n}\right)$ and $w=\left(w_{0}, w_{1}, \ldots, w_{n}\right)$ are two grid functions on $\Omega_{h}$, define the following inner product and the discrete $L^{2}$-norm

$$
\langle v, w\rangle=h \sum_{l=0}^{n} v_{l} w_{l},\|v\|=\sqrt{\langle v, v\rangle}=\left(h \sum_{l=0}^{n} v_{l}^{2}\right)^{\frac{1}{2}}
$$

Denote $W^{i, p e r}(\Omega)$ the $i$-periodic Sobolev spaces [20], [21].

Now, as in papers [2], [15], we investigate the stability and convergence analysis of the main scheme (12) in the following Theorems.

Theorem 1. Let us assume that the function $U: \Omega \rightarrow R$ be the exact solution of (1)-(3) which

$$
U \in W^{4, \infty}\left(0,+\infty ; L^{\infty}(A, B)\right) \cap L^{\infty}\left(0,+\infty ; W^{4, \infty}(A, B)\right) .
$$

Let $u$ be the solution of the difference scheme (12) and there exist constants $\chi_{l}, l=1,2,3$ such that

$$
|f(x, t, u)| \leq \chi_{1}(1+|x|+|t|+|u|), \quad|\partial f(x, t, u) / \partial x| \leq \chi_{2} \text { and }|\partial f(x, t, u) / \partial u| \leq \chi_{3} .
$$

Then, when

$$
\mu^{2}<\left\{\begin{array}{lr}
\left(1-4 \lambda_{1}\right) /(1-4 \sigma), & \text { if } \quad \rho>5 k /\left(1-4 \lambda_{1}\right), \\
{\left[\left(1-4 \lambda_{1}\right)(1+k \rho / 4)-5 / 4\right] /(1-4 \sigma),} & \text { if } \quad \rho \leq 5 k /\left(1-4 \lambda_{1}\right),
\end{array}\right.
$$

for sufficiently small $k$ there exists a constant $\chi>0$, independent of $h$ and $k$ such that

$$
\left\|u^{m+1}\right\|+\left\|u_{t}^{m}\right\|+\left\|u_{x}^{m+1}\right\| \leq \chi .
$$

Proof. We can rewrite (12) in the following form

$$
\left(\delta_{t}^{2}+(k \rho / 2) \delta_{2 t}\right)\left(1+\lambda_{1} \delta_{x}^{2}\right) u^{j}-\mu^{2} \delta_{x}^{2}\left(1+\sigma \delta_{t}^{2}\right) u^{j}+k^{2}\left(1+\lambda_{1} \delta_{x}^{2}\right) f^{j}=0 .
$$


Multiplying (15) by $\left(h \delta_{2 t} u_{l}^{j}\right)$ and summing up for $l$ from 0 to $n$, we have

$$
\begin{gathered}
\left\langle\delta_{t}^{2}\left(1+\lambda_{1} \delta_{x}^{2}\right) u^{j}, \delta_{2 t} u^{j}\right\rangle+(k \rho / 2)\left\langle\delta_{2 t}\left(1+\lambda_{1} \delta_{x}^{2}\right) u^{j}, \delta_{2 t} u^{j}\right\rangle-\mu^{2}\left\langle\delta_{x}^{2}\left(1+\sigma \delta_{t}^{2}\right) u^{j}, \delta_{2 t} u^{j}\right\rangle+ \\
k^{2}\left\langle\left(1+\lambda_{1} \delta_{x}^{2}\right) f^{j}, \delta_{2 t} u^{j}\right\rangle=0 .
\end{gathered}
$$

By using the preliminary definitions and Lemmas in [2], we have

$$
\begin{gathered}
\left\|\delta_{t}^{+} u^{j}\right\|^{2}-\left\|\delta_{t}^{-} u^{j}\right\|^{2}-\lambda_{1}\left(\left\|\delta_{x}^{+} \delta_{t}^{+} u^{j}\right\|^{2}-\left\|\delta_{x}^{+} \delta_{t}^{-} u^{j}\right\|^{2}\right)+(k \rho / 2)\left(\left\|\delta_{2 t} u^{j}\right\|^{2}-\lambda_{1}\left\|\delta_{x}^{+} \delta_{2 t} u^{j}\right\|^{2}\right)+ \\
\sigma \mu^{2}\left(\left\|\delta_{x}^{+} u^{j+1}\right\|^{2}-\left\|\delta_{x}^{+} u^{j-1}\right\|^{2}\right)+(1-2 \sigma)\left(\left\langle\delta_{x}^{+} u^{j+1}, \delta_{x}^{+} u^{j}\right\rangle-\left\langle\delta_{x}^{+} u^{j}, \delta_{x}^{+} u^{j-1}\right\rangle\right) \leq \\
k^{2}\left[(1 / 2)\left(\left\|\delta_{2 t} u^{j}\right\|^{2}+\left\|\delta_{x}^{+} \delta_{2 t} u^{j}\right\|^{2}\right)+4 \chi_{1}^{2}\left(1+\left\|u^{j}\right\|^{2}\right)+4 \lambda_{1}^{2}\left(\chi_{2}^{2} h^{2}(b-a)+\chi_{3}^{2}\left\|\delta_{x}^{+} u^{j}\right\|^{2}\right)\right] .
\end{gathered}
$$

By summing up (17) for $j$ from 1 to $m$ and using $\delta_{t}^{-} u^{j}=\delta_{t}^{+} u^{j-1}$, we have

$$
\begin{aligned}
& \left\|\delta_{t}^{+} u^{m}\right\|^{2}-\lambda_{1}\left\|\delta_{x}^{+} \delta_{t}^{+} u^{m}\right\|^{2}+\left((k \rho / 2)-\left(k^{2} / 2\right)\right) \sum_{j=1}^{m}\left\|\delta_{2 t} u^{j}\right\|^{2}-\left(\left(k \rho \lambda_{1} / 2\right)+\left(k^{2} / 2\right)\right) \sum_{j=1}^{m}\left\|\delta_{x}^{+} \delta_{2 t} u^{j}\right\|^{2}+ \\
& \sigma \mu^{2}\left(\left\|\delta_{x}^{+} u^{m+1}\right\|^{2}+\left\|\delta_{x}^{+} u^{m}\right\|^{2}\right)+(1-2 \sigma) \mu^{2}\left\langle\delta_{x}^{+} u^{m+1}, \delta_{x}^{+} u^{m}\right\rangle \leq\left\|\delta_{t}^{-} u^{1}\right\|^{2}-\lambda_{1}\left\|\delta_{x}^{+} \delta_{t}^{+} u^{0}\right\|^{2}+\sigma \mu^{2}\left(\left\|\delta_{x}^{+} u^{0}\right\|+\left\|\delta_{x}^{+} u^{1}\right\|\right)+ \\
& +(1-2 \sigma) \mu^{2}\left\langle\delta_{x}^{+} u^{1}, \delta_{x}^{+} u^{0}\right\rangle+k^{2}\left[4 m \chi_{1}^{2}\left(1+\left\|u^{j}\right\|^{2}\right)+4 \chi_{1}^{2} \sum_{j=1}^{m}\left\|u^{j}\right\|^{2}+4 \lambda_{1}^{2}\left(m \chi_{2}^{2} h^{2}(b-a)+\chi_{3}^{2} \sum_{j=1}^{m}\left\|\delta_{x}^{+} u^{j}\right\|^{2}\right)\right] .
\end{aligned}
$$

By using the identity defined in [2], we have

$$
\begin{aligned}
& \left\|\delta_{t}^{+} u^{m}\right\|^{2}-\left(\lambda_{1}+\left((1-2 \sigma) \mu^{2} / 2\right)\right)\left\|\delta_{x}^{+} \delta_{t}^{+} u^{m}\right\|^{2}+\left((k \rho / 2)-\left(k^{2} / 2\right)\right) \sum_{j=1}^{m}\left\|\delta_{2 t} u^{j}\right\|^{2}-\left(\left(k \rho \lambda_{1} / 2\right)+\right. \\
& \left.\left(k^{2} / 2\right)\right) \sum_{j=1}^{m}\left\|\delta_{x}^{+} \delta_{2 t} u^{j}\right\|^{2}+\left(\mu^{2} / 2\right)\left(\left\|\delta_{x}^{+} u^{m+1}\right\|^{2}+\left\|\delta_{x}^{+} u^{m}\right\|^{2}\right) \leq\left\|\delta_{t}^{-} u^{1}\right\|^{2}+\sigma\left\|\delta_{x}^{+} u^{0}\right\|+((1-2 \sigma) / 2) \times \\
& \left(\left\|\delta_{x}^{+} u^{1}\right\|^{2}+\left\|\delta_{x}^{+} u^{0}\right\|^{2}-\left\|\delta_{x}^{+} \delta_{t}^{+} u^{0}\right\|^{2}\right)+k^{2}\left[4 m \chi_{1}^{2}+4 \chi_{1}^{2} \sum_{j=1}^{m}\left\|u^{j}\right\|^{2}+4 \lambda_{1}^{2}\left(m \chi_{2}^{2} h^{2}(b-a)+\chi_{3}^{2} \sum_{j=1}^{m}\left\|\delta_{x}^{+} u^{j}\right\|^{2}\right)\right] .
\end{aligned}
$$

We know that $\delta_{t}^{-} u^{1}=\delta_{t}^{+} u^{0}$, by help of Lemma 2 in [2] the fourth term on the left hand side of (18) can be written as

$$
\begin{aligned}
& {\left[\left(1-4 \lambda_{1}\right)-(1-4 \sigma) \mu^{2}-2 \varepsilon \mu^{2}\right]\left\|\delta_{t}^{+} u^{m}\right\|^{2}+\left(\left(k \rho\left(1-4 \lambda_{1}\right) / 2\right)-\left(5 k^{2} / 2\right)\right) \sum_{j=1}^{m}\left\|\delta_{2 t} u^{m}\right\|^{2}+\varepsilon \mu^{2}\left(\left\|\delta_{x}^{+} u^{m+1}\right\|^{2}+\right.} \\
& \left.\left\|\delta_{x}^{+} u^{m}\right\|^{2}\right) \leq\left\|\delta_{t}^{+} u^{0}\right\|+\sigma\left\|\delta_{x}^{+} u^{0}\right\|+((1-2 \sigma) / 2)\left(\left\|\delta_{x}^{+} u^{1}\right\|^{2}+\left\|\delta_{x}^{+} u^{0}\right\|^{2}\right)+k^{2}\left\{\left\|\delta_{t}^{+} u^{0} / k\right\|^{2}+\left(\sigma / \mu^{2}\right)\left\|\delta_{x}^{+} u^{0} / h\right\|^{2}+\right. \\
& \left.\left((1-2 \sigma) / \mu^{2}\right)\left(\left\|\delta_{x}^{+} u^{1} / h\right\|^{2}+\left\|\delta_{x}^{+} u^{0} / h\right\|^{2}\right)+\left[4 m \chi_{1}^{2}+4 \chi_{1}^{2} \sum_{j=1}^{m}\left\|u^{j}\right\|^{2}+4 \lambda_{1}^{2}\left(m \chi_{2}^{2} h^{2}(b-a)+\chi_{3}^{2} \sum_{j=1}^{m}\left\|\delta_{x}^{+} u^{j}\right\|^{2}\right)\right]\right\} .
\end{aligned}
$$

where $\varepsilon$ is a sufficiently small coefficient. In the case $\left(\rho\left(1-4 \lambda_{1}\right)-5 k\right) / 2>0$, i.e., $\rho>5 k /\left(1-4 \lambda_{1}\right)$, as $\varepsilon$ can be sufficiently small, we need that $\mu^{2}<\left(1-4 \lambda_{1}\right) /(1-4 \sigma)$. In other case, as 


$$
\left\|\delta_{2 t} u^{m}\right\|^{2}=\left\|\delta_{t}^{+} u^{j}+\delta_{t}^{-} u^{j}\right\|^{2} \leq\left\|\delta_{t}^{+} u^{j}\right\|^{2}+\left\|\delta_{t}^{-} u^{j}\right\|^{2}
$$

holds, we require that $\mu$ satisfy the following condition (for $j=m$ )

$$
\left(1-4 \lambda_{1}\right)-(1-4 \sigma) \mu^{2}+\left[k \rho\left(1-4 \lambda_{1}\right)-5 k^{2}\right] / 2>0 .
$$

Then we have $\mu^{2}<\left[\left(1-4 \lambda_{1}\right)(1+k \rho / 4)-5 k^{2} / 4\right] /(1-4 \sigma)$. Therefore we proved the stability condition. Now we can easily prove that $\left\|\delta_{t}^{+} u^{0} / k\right\|,\left\|\delta_{x}^{+} u^{0} / h\right\|$ and $\left\|\delta_{x}^{+} u^{1} / h\right\|$ are all bounded, consequently, all the terms on the right hand side of (20) have upper bound.

Therefore we confirm that all the terms on the right hand side of (20) are bounded. From the above notations we conclude that all the coefficients on the left hand side of (20) are positive when $\rho>5 k /\left(1-4 \lambda_{1}\right)$. Hence from Lemma 2 in [2] in both cases we obtain that $\left\|\delta_{t}^{+} u^{m}\right\|+\mu\left\|\delta_{x}^{+} u^{m+1}\right\| \leq k \chi$, i.e, $\left\|u_{t}^{m}\right\|+\mu\left\|u_{x}^{m+1}\right\| \leq \chi$, under the stability condition. The relation $u^{m+1}=u^{0}+\sum_{j=1}^{m} \delta_{t}^{+} u^{j}$, gives that $\left\|u^{m+1}\right\| \leq\left\|u^{0}\right\|+m k \chi$. The proof is completed.

Theorem 2. Suppose that the solution $u(x, t)$ of (1)-(3) is sufficiently regular and the assumptions of Theorem (1) are valid. For $k$ sufficiently small, the solution of the spline difference scheme (12) converges to the solution of (1)-(3) in the discrete $L^{2}$-norm and we have

$$
\left\|(U-u)^{m+1}\right\|+\left\|(U-u)_{t}^{m}\right\|+\left\|(U-u)_{x}^{m+1}\right\| \leq \begin{cases}\chi\left(k^{2}+k^{2} h^{2}+h^{2}\right), & \text { if } 2\left(\lambda_{1}+\lambda_{2}\right)=1, \quad \lambda_{1} \neq 1 / 12 \\ \chi\left(k^{2}+k^{2} h^{2}+h^{4}\right), & \text { if } \quad \lambda_{1}=1 / 12, \quad \lambda_{2}=5 / 12 .\end{cases}
$$

\section{Numerical Illustrations}

We applied the presented schemes to the following nonlinear generalized Sine-Gordon equation. We solve following example by our $O\left(k^{2}+k^{2} h^{2}+h^{2}\right)$ method with $\left(\lambda_{1}, \lambda_{2}\right)=(1 / 6,1 / 3)$ and our $O\left(k^{2}+k^{2} h^{2}+h^{4}\right)$ method with $\left(\lambda_{1}, \lambda_{2}\right)=(1 / 12,5 / 12)$. We tabulate the computed errors in solution in our Tables and compare our results with the results in [2].

Example. Consider the nonlinear Sine-Gordon equation:

$$
u_{t t}+\rho u_{t}=u_{x x}-2 \sin u-\pi^{2} e^{-t} \cos \pi x+2 \sin \left(e^{-t}(1-\cos \pi x)\right), \quad x \in(0,2), \quad t>0,
$$

subjected to the initial conditions

$$
u(x, 0)=1-\cos \pi x, \quad u_{t}(x, 0)=-1+\cos \pi x, \quad x \in[0,2],
$$

and with Dirichlet boundary conditions

$$
u(0, t)=u(2, t)=0, \quad t \geq 0 .
$$

The exact solution for this problem is $u(x, t)=e^{-t}(1-\cos \pi x)$. 
We solve this problem with $h=0.1,0.05, k=0.01,0.005, \sigma=\rho / 6$ and different values of the parameters $\lambda_{1}$ and $\lambda_{2}$. Observed $L_{2}$ and RMS errors in the computed solution are tabulated in Tables 1-2 for different times. The results in Tables show that our $O\left(k^{2}+k^{2} h^{2}+h^{4}\right)$ method is more accurate than the methods in [2].

Table 1. Observed $L_{2}$ and RMS Errors in Our $O\left(k^{2}+k^{2} h^{2}+h^{2}\right)$ Method

\begin{tabular}{|c|c|c|c|c|c|c|}
\hline \multirow[b]{2}{*}{$t$} & \multicolumn{2}{|c|}{$h=0.1, \quad k=0.01$} & \multicolumn{2}{|c|}{$h=0.05, \quad k=0.01$} & \multicolumn{2}{|c|}{$h=0.05, \quad k=0.005$} \\
\hline & $L_{2}$ & RMS & $L_{2}$ & RMS & $L_{2}$ & RMS \\
\hline 1.0 & $2.13(-2)$ & $4.66(-3)$ & $7.61(-3)$ & $1.18(-3)$ & $7.54(-3)$ & $1.17(-3)$ \\
\hline 2.0 & $8.71(-3)$ & $1.90(-3)$ & $3.13(-3)$ & $4.89(-4)$ & $3.13(-3)$ & $4.89(-4)$ \\
\hline 3.0 & $5.79(-3)$ & $1.26(-3)$ & $2.05(-3)$ & $3.20(-4)$ & $2.03(-3)$ & $3.17(-4)$ \\
\hline 4.0 & $2.63(-3)$ & $5.74(-4)$ & $9.41(-4)$ & $1.47(-4)$ & $9.30(-4)$ & $1.45(-4)$ \\
\hline 5.0 & $1.55(-3)$ & $3.40(-4)$ & $5.53(-4)$ & $8.64(-5)$ & $5.52(-4)$ & $8.63(-5)$ \\
\hline
\end{tabular}

Table 2. Observed $L_{2}$ and RMS Errors in Our $O\left(k^{2}+k^{2} h^{2}+h^{4}\right)$ Method

\begin{tabular}{c|c|c|c|c|c|c}
\hline \hline \multirow{2}{*}{$t$} & \multicolumn{2}{|c|}{$h=0.1, \quad k=0.01$} & \multicolumn{2}{c|}{$h=0.05, \quad k=0.01$} & \multicolumn{2}{l}{$h=0.05, \quad k=0.005$} \\
\cline { 2 - 7 } & $L_{2}$ & RMS & $L_{2}$ & RMS & $L_{2}$ & RMS \\
\hline 1.0 & $7.12(-5)$ & $1.55(-5)$ & $3.99(-5)$ & $6.24(-6)$ & $2.98(-6)$ & $4.65(-7)$ \\
\hline 2.0 & $2.88(-5)$ & $6.29(-6)$ & $1.74(-5)$ & $2.71(-6)$ & $1.50(-6)$ & $2.35(-7)$ \\
\hline 3.0 & $1.90(-5)$ & $4.14(-6)$ & $1.09(-5)$ & $1.71(-6)$ & $8.72(-7)$ & $1.36(-7)$ \\
\hline 4.0 & $8.77(-6)$ & $1.91(-6)$ & $5.04(-6)$ & $7.87(-7)$ & $3.98(-7)$ & $6.21(-8)$ \\
\hline 5.0 & $5.10(-6)$ & $1.11(-6)$ & $2.99(-6)$ & $4.67(-7)$ & $2.44(-7)$ & $3.81(-8)$ \\
\hline \hline
\end{tabular}

\section{Conclusion}

In this article, we constructed a three time-level spline-difference scheme for the one-dimensional generalized sine-Gordon equation. We proved the stability and convergence of the developed schemes by energy method for our scheme. We prove that our presented schemes are unconditionally stable. To examine the accuracy and efficiency of the proposed algorithm, we give one numerical examples. These computational results show that our proposed algorithm is effective and accurate in comparison with [2].

\section{References}

[1] Dehghan, M., \& Shokri, A. (2008). A numerical method for one-dimensional nonlinear sine-Gordon equation using collocation and radial basis functions. Number Methods Partial Differential Eq., 24, 
687-698.

[2] Cui, M. (2009). Fourth-order compact scheme for the one-dimensional sine-gordon equation. Numer. Methods Partial Differential Eq., 25, 685-711.

[3] Eisenhart, L. P. (1960). A Treatise on the Differential Geometry of Curves and Surfaces. NewYork.

[4] Bäcklund, A. V. (1876). Ueber flächen transformation en. Mathematische Annalen, 9, 297-320.

[5] Bianchi, L. (1910). Vorlesungenüber Differential Geometrie. Teubner, Leibzig.

[6] Dodd, R. K., Eilbeck, J. C., Gibbon, J. D., \& Morris, H. C. (1982). Solitons in Nonlinear Wave Equations. New York: Academic Press.

[7] Drazin, P. J., \& Johnson, R. S. (1989). Solitons: An Introduction. Cambridge, UK: Cambridge University Press.

[8] Tabor, M. (1989). The Sine-Gordon Equation. 7.5.b in Chaos and Integrability in Nonlinear Dynamics: An Introduction. Wiley, New York

[9] Brenner, P. (2011). Lipschitz continuity of the scattering operator for nonlinear Klein-Gordon equations. Applied Computational Mathematics, 10(1), 213-241.

[10] Batiha, B., Noorani, M. S. M., \& Hashim, I. (2007). Numerical solution of sine-Gordon equation by variational iteration method. Physics Letters A, 370, 437-440.

[11] Dehghan, M., \& Mirzaei, D. (2008). The boundary integral equation approach for numerical solution of the one-dimensional sine-gordon equation. Numer. Methods for Partial Differential Equations, 24, 1405-1415.

[12] Dehghan, M. \& Shokri, A. (2008). A numerical method for solution of the two-dimensional sine-gordon equation using the radial basis functions. Mathematics and Computers in Simulation, 79, 700-715.

[13] Fabian, A. L., Kohl, R., \& Biswas, A. (2009). Perturbation of topological solitons due to sine-gordon equation and its type. Communicationsin Nonlinear Science and Numerical Simulation, 14, 1227-1244.

[14] Ben-Yu, G., Pascual, P. J., Rodriguez, M. J., \& Vázquez, L. (1986). Numerical solution of the sine-gordon equation. Applied Mathematics and Computation, 18, 1-14.

[15] Sheng, Q., Khaliq, A. Q. M., \& Voss, D. A. (2005). Numerical simulation of two-dimensional sine-gordon solitons via a split cosine scheme. Mathematics and Computers in Simulation, 68, 355-373.

[16] Bratsos, A. G. (2008). A numerical method for the one-dimensional sine-gordon equation. Numerical Methods for Partial Differential Equations, 24, 833-844.

[17] Mohammadi, R. (2014). An exponential spline solution of nonlinear Schrödinger equations with constant and variable coefficients. Computer Physics Communications, 185, 917-932.

[18] Mohammadi, R. (2014). Sextic B-spline collocation method for solving euler-bernoulli beam models. Appl. Math. Comput., 241, 151-166.

[19] Mohammadi, R. (2015). Quintic B-spline collocation approach for solving generalized black-scholes equation governing option pricing. Computers and Mathematics with Applications, 69, 777-797.

[20] Adams, R. A. (1978). Sobolev Spaces. Academic Press.

[21] Atkinson, K., \& Han, W. (2005). Theoretical Numerical Analysis, A Functional Analysis Framework. Springer.

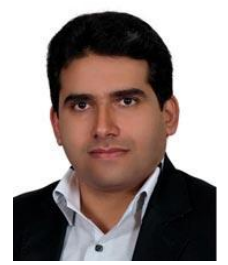

Reza Mohammadi is an assistant professor of applied mathematics in the Department of Mathematics, University of Neyshabur, Neyshabur, Iran, since 2010. He received his Ph.D. degree in applied mathematics (numerical analysis) from Iran University of Science and Technology in 2010. Now he is vice-chancellor of student and cultural in University of Neyshabur. His research interests are numerical analysis, numerical solution of ordinary and partial differential equations, spline approximations and financial mathematics. 